\title{
Element Diffusion in Pulsating Variables
}

\author{
Joyce Ann Guzik
}

\author{
Los Alamos National Laboratory, Los Alamos, New Mexico, USA
}

\begin{abstract}
Can pulsation studies yield information on the operation of element diffusion in stars? Can diffusion explain unusual properties of pulsating variables? Element diffusion theory and recent research relevant to these questions will be reviewed, with emphasis on the Sun and $\delta$ Scuti stars. Highdegree solar p-modes that are sensitive to helium ionization support a reduced convection zone helium abundance consistent with that expected from diffusion. Intermediate-degree p-modes can be used to probe the structure of the convection zone base and constrain possible diffusion-produced composition gradients. $\delta$ Scuti variables have shallow convection zones and relatively short diffusion timescales. Helium diffusion may explain unusual period ratios, influence period changes, and affect the amplitudes and light curve shapes of $\delta$ Scuti stars.
\end{abstract}

\section{Introduction}

There are several ways in which pulsation studies may help us understand diffusion, or diffusion studies may help to explain pulsation observations. For the Sun, white dwarfs, rapidly oscillating Ap stars, and possibly $\delta$ Scuti stars, the rich spectrum of nonradial pand/or g-modes can be used to probe diffusion-produced stratification and composition gradients. Diffusion of helium and heavier elements can affect pulsation driving and instability strip boundaries. Diffusion calculations may provide indirect information on competing mechanisms that could affect pulsation, such as meridional circulation, turbulence, horizontal shear, convection, mass loss, and accretion. Observations of period and amplitude changes, light curve shapes, or episodic pulsation, combined with theoretical modeling may help to constrain/infer diffusion rates.

A number of recent papers discuss diffusion and pulsation for several types of variable stars. Guzik and Cox (1992), Vorontsov et al. (1992), and Dziembowski et al. (1992) use solar p-modes to infer a convection zone helium mass fraction $Y \cong 0.23-0.24$, less than the initial abundance needed to match the solar luminosity by exactly the amount expected from diffusion. For DB white dwarfs, Bradley (1992) finds that g-mode period spacings can be used to find the helium layer mass and probe the He/C transition region. Matthews (1991) suggests that p-modes be used to probe the structure of roAp stars, where diffusion plays a role in observed abundance anomalies. For $\delta$ Scuti stars, Cox et al. (1984) suggest that helium diffusion is responsible for the high $1 \mathrm{H} / \mathrm{F}$ period ratio of VZ Cnc; Poretti and 
Antonello (1988) suggest that helium diffusion could produce the unusual light curves of some large-amplitude $\delta$ Scuti stars. Saez et al. (1981) propose that a combination of helium diffusion, radiative levitation, and mixing would maintain enough helium in the driving region to allow pulsation and yet maintain mild abundance anomalies observed in $\delta$ Del stars. For RR Lyrae stars, Michaud et al. (1983) suggest that helium diffusion may affect the instability strip blue edge, where these stars have smaller envelope convection zones, and that radiative levitation may produce $\mathrm{Ca}$ abundance anomalies that should be taken into account in $\mathrm{Z}$ determinations from Ca II lines. Cox et al. (1992) propose that radiative levitation of iron provides the $\kappa$-effect pulsation driving for $\beta$ Cephei stars.

\section{Diffusion calculations}

Michaud and Proffitt (1992) review the status of diffusion calculation methods and applications to solar and stellar evolution. Here we will describe the method of Burgers (1969). The equations of diffusion, heat flow, no net mass flow, and no net current for each ionic species and the electron are given by:

$$
\begin{gathered}
\nabla p_{i}-\frac{\rho_{i}}{\rho} \nabla p-n_{i} q_{i} E=\sum_{j} K_{i j}\left(w_{j}-w_{i}\right)+\sum_{j} K_{i j} z_{i j} \frac{m_{j} r_{i}-m_{i} r_{j}}{m_{i}+m_{j}} \\
\frac{5}{2} n_{i} k \nabla T=-\frac{5}{2} \sum_{j \neq i} K_{i j} z_{i j} \frac{m_{j}}{m_{i}+m_{j}}\left(w_{j}-w_{i}\right)-\frac{2}{5} K_{i i} z_{i j}^{\prime \prime} r_{i} \\
-\sum_{j \neq i} \frac{K_{i j}}{\left(m_{i}+m_{j}\right)^{2}}\left(3 m_{i}^{2}+m_{j}^{2} z_{i j}^{\prime}+0.8 m_{i} m_{j} z_{i j}^{\prime \prime}\right) r_{i}+\sum_{j \neq i} \frac{K_{i j} m_{i} m_{j}}{\left(m_{i}+m_{j}\right)^{2}}\left(3+z_{i j}^{\prime}-0.8 z_{i j}^{\prime \prime}\right) r_{j} \\
\sum A_{i} n_{i} w_{i}=0 ; \quad \sum Z_{i} n_{i} w_{i}=0
\end{gathered}
$$

These equations are solved for the unknown diffusion velocities $w_{i}$, the residual heat flow vectors $r_{i}$, and the electric field $E$. The first two terms on the left hand side of the diffusion equation can be rewritten using the equation of hydrostatic equilibrium and the ideal gas law for a given ion species as:

$$
\frac{1}{n_{i}}\left(\nabla p_{i}-\frac{\rho_{i}}{\rho} \nabla p\right)=-A_{i} m_{H} g-k T \frac{d \ln T}{d r}-k T \frac{d \ln n_{i}}{d r}
$$

The first two right-hand terms including the gravitational acceleration and the temperature gradient are considered the driving terms for "gravitational" diffusion. Radiative levitation can be included by subtracting from the gravitational acceleration the radiative acceleration on the ion species. The last term containing the composition gradient drives "chemical" diffusion. "Thermal" diffusion is accounted for by including the heat flow equations (2). The resistance coefficients $K_{i j}$ and $z_{i j}, z_{i j}{ }^{\prime}$ and $z_{i j}{ }^{\prime \prime}$ are functions of the collision integrals. The best available analytical fits to these integrals are given by Paquette et al. (1986).

The assumptions in this method are: complete ionization; no magnetic fields; all species have Maxwellian velocity distributions at the same temperature; thermal velocities are much greater than diffusion velocities; collisions are dominated by classical interactions between 
point particles; and the plasma is considered a dilute gas, i.e. the plasma parameter $\Lambda<<1$, and the Boltzmann equation is rigorously valid. For stellar interiors, $\Lambda \sim 1$, in the intermediate regime between weak and strong coupling, and the last two assumptions are questionable. Paquette et al. (1986) discuss the potential uncertainties in this treatment due to the breakdown of these assumptions.

The diffusion velocities $w_{i}$ are used to solve the species equation of motion

$$
\frac{\partial n_{i}}{\partial t}=-\frac{1}{r^{2}} \frac{\partial}{\partial r}\left(r^{2} n_{i} w_{i}\right)
$$

This equation is discretized to 1 st order in time and 2 nd order in space, and solved by standard matrix techniques. Iben and MacDonald (1985) describe their solution method and implementation in the Iben stellar evolution code.

\section{Diffusion in the Sun}

Solar evolution calculations including diffusion (Cox, Guzik and Kidman, 1989; Proffitt and Michaud, 1991) show that diffusion can reduce the solar convection zone helium mass fraction by $\sim 10 \%$, and the convection zone $Z$ by $5-10 \%$ during the Sun's 4.5 billion year lifetime. Guzik and Cox (1992) find that solar p-modes of degree $l=300-600$ have large weight functions in the helium ionization region between 50,000 and $300,000 \mathrm{~K}$, and are quite sensitive to the helium abundance. Table I gives the sensitivity of these p-mode frequencies to a reduction of 0.03 in convection zone helium mass fraction from the initial $Y \cong 0.27$ required to match the solar luminosity, and the effects on the observed minus calculated frequencies. The sensitivity for many $l=300-600$ modes is $5-8 \mu \mathrm{Hz}$, much larger than the observational uncertainties of $0.2-0.6 \mu \mathrm{Hz}$. The $\mathrm{O}-\mathrm{C}$ frequencies are much improved by the reduced helium for exactly those frequencies that show sensitivity, supporting the diffusion results.

Diffusion produces a rather steep $\mathrm{Y}$ (and Z) gradient just below the convection zone. Proffitt and Michaud (1991) show that turbulence, possibly induced by convective overshoot or meridional circulation, in the amount required to account for the solar lithium depletion, would smooth this gradient and reduce the amount of helium settling from the convection zone. The helium diffusion composition profiles of Cox, Guzik and Kidman (1989) and of Proffitt and Michaud (1991) for different turbulence treatments are shown in Fig. 1. We discuss next how intermediate-degree p-modes $(l=5-30)$, with large weight functions near the convection zone bottom, can be used to constrain the shape of a diffusion-produced composition gradient.

Figures $2 a, b$, and $c$ show the observed minus calculated $(\mathrm{O}-\mathrm{C}) \mathrm{p}$-mode frequencies versus frequency for degrees $l=5-60$ for several solar models. Lines connect modes with the same degree $l$. The solar evolution and oscillation procedures are described in Guzik and Cox (1991a). The observations (Libbrecht et al., 1990) have quoted uncertainties of less than $0.1 \mu \mathrm{Hz}$. The model of Fig. 2a has no diffusion, and $(Y, Z)=(0.267,0.02)$. The model of Fig. $2 b$ includes $Y$ and $Z$ diffusion, but no turbulence, and so has a steep composition gradient below the convection zone. The initial $\mathrm{Y}$ required to match the solar luminosity is 0.264 , and the convection zone $(\mathrm{Y}, \mathrm{Z})$ after $4.5 \mathrm{Gyr}$ is $(0.236,0.019)$. The model of Fig. $2 c$ includes diffusion, but has a $\mathrm{Y}$ (and $\mathrm{Z}$ ) composition gradient that is linear 
Table I Sensitivity of p-mode frequencies to solar convection zone helium abundance

\begin{tabular}{|c|c|c|c|c|c|}
\hline \multicolumn{2}{|c|}{ p-mode } & \multirow{2}{*}{$\begin{array}{c}\text { Sensitivity }(\mu \mathrm{Hz}) \\
v(0.27)-v(0.24)\end{array}$} & \multicolumn{2}{|c|}{ O-C Frequency $(\mu \mathrm{Hz})$} & \multirow{2}{*}{$\begin{array}{c}\text { Observational } \\
\text { Uncertainty }(\mu \mathrm{Hz})\end{array}$} \\
\hline 1 & $\mathrm{n}$ & & $\mathrm{Y}_{\mathrm{cz}}=0.27$ & $Y_{c z}=0.24$ & \\
\hline \multirow[t]{4}{*}{300} & 1 & 5.2 & -2.3 & 2.9 & 0.3 \\
\hline & 2 & 6.1 & -4.7 & 1.4 & 0.2 \\
\hline & 3 & 2.3 & -2.8 & -0.4 & 0.2 \\
\hline & 4 & 0.1 & -0.2 & -0.1 & 0.3 \\
\hline \multirow[t]{3}{*}{395} & 1 & 5.7 & -4.6 & 1.1 & 0.6 \\
\hline & 2 & 7.0 & -9.2 & -2.2 & 0.3 \\
\hline & 3 & 7.5 & .10 .7 & -3.2 & 0.4 \\
\hline \multirow[t]{4}{*}{400} & 0 & 0.0 & 6.6 & 6.6 & 0.6 \\
\hline & 1 & 5.8 & -5.4 & 0.4 & 0.3 \\
\hline & 2 & 7.2 & -8.8 & -1.6 & 0.2 \\
\hline & 3 & 8.0 & -9.3 & -1.3 & 0.2 \\
\hline \multirow[t]{2}{*}{600} & 1 & 4.7 & .11 .7 & -7.0 & 0.8 \\
\hline & 2 & 7.0 & -12.4 & -5.4 & 0.3 \\
\hline
\end{tabular}

Observations are the most accurate of either Korzennik (1990) or Libbrecht et al. (1990).

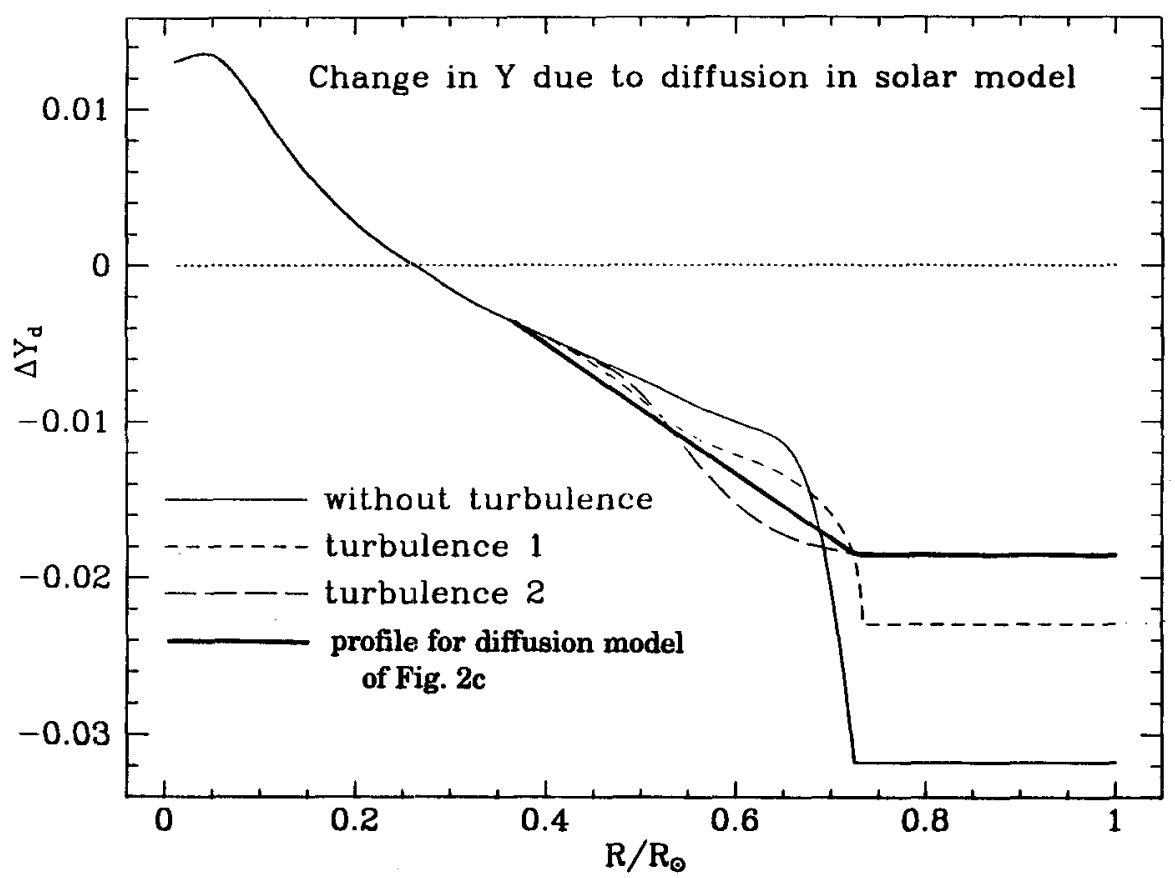

Figure 1 Change in Y due to diffusion in solar models (Proffitt and Michaud, 1991). 

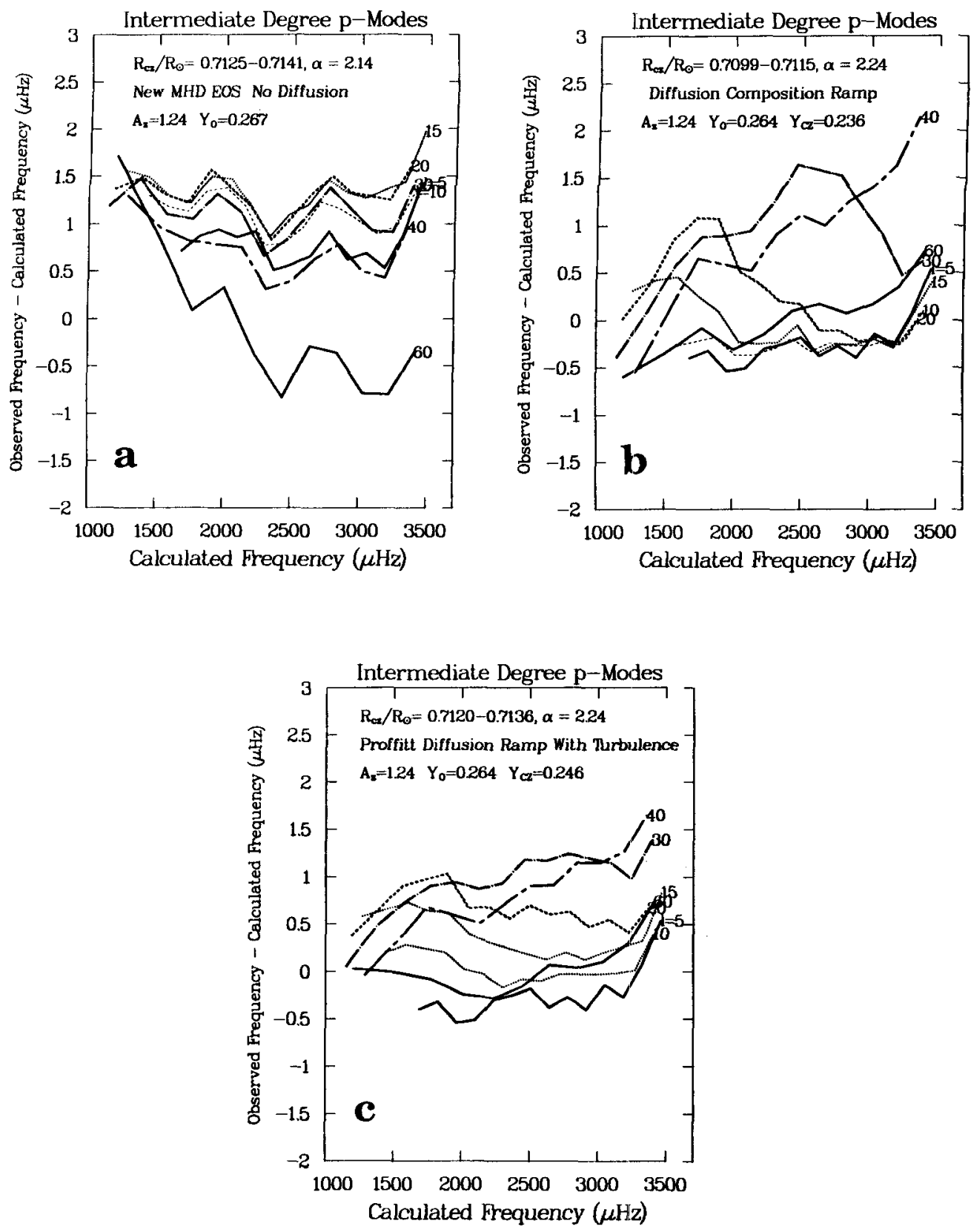

Figure 2 Observed minus calculated vs. calculated p-mode frequencies for solar models without diffusion (a); with diffusion (b); and with the diffusion-produced $\mathrm{Y}$ (and $\mathrm{Z}$ ) composition gradient below the convection zone modified by turbulence (c) as in Fig. 1 . Lines connect modes of the same degree $l$. 
with respect to radius, as indicated in Fig. 1, intermediate between the two turbulence models. Turbulence decreases the amount of $Y$ diffusion; $Y=0.246$ in the convection zone.

The $\mathrm{O}-\mathrm{C}$ fits in these figures have already been partially optimized (to be generally flat rather than sloping versus frequency), by adjusting the radius of the convection zone bottom via a small change in the mixing length/pressure scale height ratio for the inward integration in our fine-zone model building code. (This procedure preserves solar luminosity and radius). In this way, $\mathrm{p}$-mode observations can be used to find the radius of the convection zone base $\left(0.712 \pm 0.002 \mathrm{R}_{\odot}\right.$; see review by A. N. Cox $)$. The convection zone depth could be adjusted properly in the evolution calculations to agree with the indications from the p-mode observations, by a very small $(\$ 1 \%)$ adjustment in the opacity below the convection zone (within the opacity uncertainties), which would in turn affect the mixing length needed to match the solar radius.

To interpret these plots, a few comments are necessary: The modes of degree $l=40$ and 60 , as well as the low-frequency modes for smaller $l$, sample the solar structure only within the convection zone, so the O-C differences may be attributed in part to inaccuracies in the solar model structure due to convection treatment. At low frequency $(\sim 1000 \mu \mathrm{Hz})$, all of the plotted modes have some sensitivity to the helium ionization region. The ability of the $l=5-30$ modes to sense the structure below the convection zone increases with increasing frequency.

A careful study and comparison of these plots reveal: 1) The O-C values at low frequency are $\sim 1.5 \mu \mathrm{Hz}$ positive for the no-diffusion model (Fig. 2a) with highest $\mathrm{Y}_{\mathrm{C}}$, slightly negative for the diffusion model (Fig. $2 b$ ) with $Y_{c z}=0.236$, and slightly positive for turbulence model (Fig. 2c) with $Y_{c z}=0.236$. Thus a convection zone helium abundance between 0.236 and 0.246 optimizes the low-frequency O-C's. 2) For high-frequency $l=5$ 20 modes with considerable weight below the convection zone, the O-C's of the diffusion model (Fig. 2b) are quite flat with frequency, and coincide for different degrees $l$. The $\mathrm{O}$ C's of the no-diffusion model (Fig. 2a) vary by about a microhertz over the frequency range considered, and are somewhat dispersed with changing degree $l$. The $\mathrm{O}-\mathrm{C}$ fit of the diffusion model (Fig. 2b) is favored. 3) For the O-C's of the model with a composition gradient modified by turbulence (Fig. 2c), the O-C curves are fairly flat in frequency, but are dispersed vertically with respect to $l$. Thus the $\mathrm{O}-\mathrm{C}$ fit for the diffusion model with a steep composition profile is favored over that of the model with turbulence.

\section{Diffusion in $\delta$ Scuti Stars}

Cox et al. (1984) suggest that envelope helium depletion could be responsible for the large observed 1st overtone/fundamental period ratio $(0.801)$ of the $\delta$ Scuti star VZ Cnc. Breger (1990) lists two Pop I $\delta$ Scuti stars, VZ Cnc, and DY Her with reported period decreases $1 / \mathrm{P} \mathrm{dP} / \mathrm{dt}=-13 \cdot 10^{-8} / \mathrm{yr}$ and $-6 \cdot 10^{-8} / \mathrm{yr}$, respectively. This is surprising, as $\delta$ Scuti stars are presumed to evolve across the instability strip from blue to red, resulting in period increases. Could helium diffusion, which would make a star more centrally concentrated, causing the period to decrease, offset the period increase expected from evolution? To answer this question, a $2 \mathrm{M}_{\odot}$ model was evolved from the zero-age main sequence across the $\delta$ Scuti instability strip, including diffusion. The calculations show that helium (and heavier elements, unless radiative levitation is included) diffuse from the upper $10^{-4} \mathrm{M}_{\odot}$ 
$(\mathrm{T}<300,000 \mathrm{~K})$ in $\sim 10^{8} \mathrm{yr}$. This implies that maintaining a nearly Pop. I envelope composition would require some mixing mechanism, or mass loss rates of the order $10^{-12}$ $\mathrm{M} \odot / \mathrm{yr}$.

The evolution timescale across the instability strip is $\sim 5 \cdot 10^{6} \mathrm{yr}$, more than an order of magnitude less than the diffusion timescale. Even if the timescales were comparable, pulsation calculations including a helium-depleted envelope show that the period decrease expected due to diffusion is only $\sim 20 \%$ of the period increase resulting from evolution, so diffusion cannot be responsible for the reported period decreases (Guzik and Cox, 1991b).

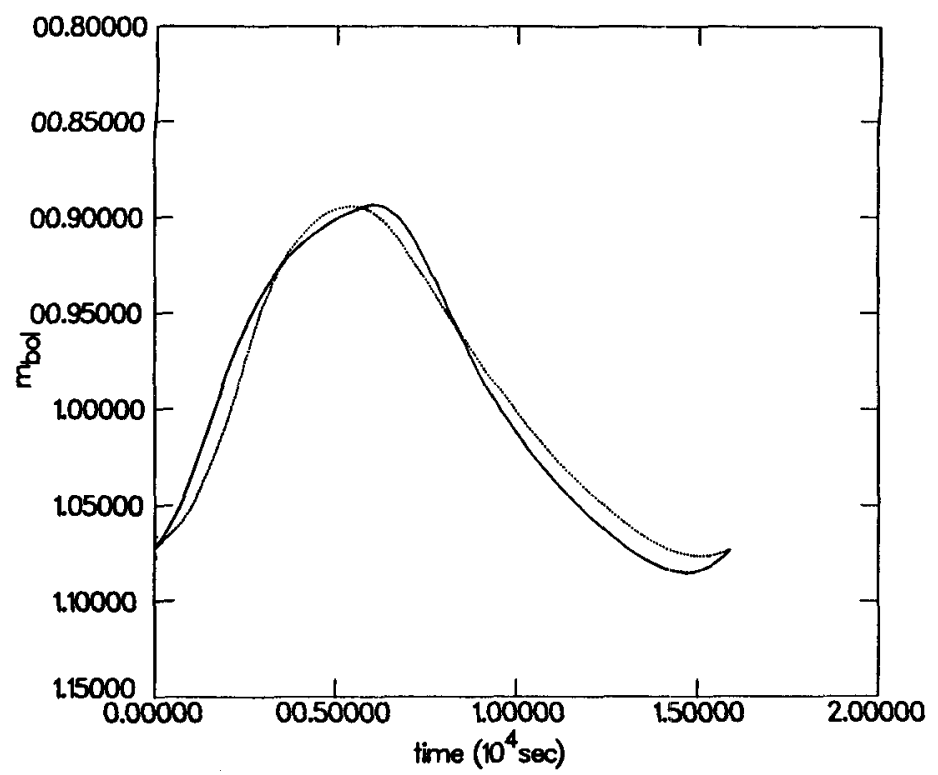

Figure 3 Bolometric magnitude of limiting amplitude solutions for $\delta$ Scuti models with envelope helium abundance decreased to $\mathrm{Y}=0.10$ for $\mathrm{T} \$ 100,000 \mathrm{~K}$ (dotted line) and $\mathrm{T} \$ 300,000 \mathrm{~K}$ (solid line). These curves show how helium diffusion could alter the light curve shapes of $\delta$ Scuti stars.

Poretti and Antonello (1988), and Antonello, Poretti, and Stellingwerf (1988) proposed, based on analysis of one-zone models, that the unusual light curves of the high-amplitude $\delta$ Scuti stars V1719 Cyg, V798 Cyg, and V974 Oph can be explained by helium diffusion. The light curves of these stars have descending branches that are steeper than the rising branch. To test this hypothesis, limiting amplitude solutions were calculated for 60-zone radiative $1.8 \mathrm{M}_{\odot} \delta$ Scuti models with and without reduced envelope helium abundances. A decrease in the helium abundance from $Y=0.28$ to $Y=0.10$ for temperatures $\$ 100,000 \mathrm{~K}$ decreases the radial velocity amplitude from $40 \mathrm{~km} / \mathrm{sec}$ to nearer the observed value of $\sim 10$ $\mathrm{km} / \mathrm{sec}$, but the light curves are still steeper on the rising branch (Fig. 3). When the helium depletion was extended to $200,000 \mathrm{~K}$, the light curve became less steep on the rising branch, and steeper on the descending branch. Extending the helium depletion to 300,000 $\mathrm{K}$ further enhances this effect (Fig. 3). The calculated light curve shapes do not quite match those observed for the variables listed above, but the approach appears promising, 
and additional calculational tests are in pregress. We also nore that if the helium abundance is reduced too much (to $Y<0.1$ ), or for too large a portion of the envelope, the heliumionization driving is weakened and the models stop pulsating.

\section{Conclusions}

Pulsation studies are useful to constrain/infer the effects of diffusion, and diffusion may be responsible for some observed properties of pulsating variables. For the Sun, high-degree p-modes support the convection zone helium abundance predicted by diffusion calculations, and intermediate-degree p-modes can be used to determine the convection zone depth and constrain diffusion-produced composition gradients. For $\delta$ Scuti stars, helium diffusion can have observable effects on period ratios, period changes, light curve shapes, and amplitudes.

\section{References:}

Antonello, E., Poretti, E., and Stellingwerf, R. F., 1988, in: Multimode Stellar Pulsations, eds. G. Kovacs, L. Szabados, and B. Szeidl, Kultura, Budapest.

Bradley, P., 1992, "The Potential of Asteroseismology of DB White Dwarfs," these proceedings.

Breger, M., 1990, in: Confrontation Between Stellar Evolution and Pulsation, Bologna, Italy, May, 1990.

Burgers, J. M., 1969, Flow Equations for Composite Gases, Academic Press, New York.

Cox, A. N., 1992, "Interpretations of Solar Oscillations," these proceedings.

Cox, A. N., Guzik, J. A., and Kidman, R. B., 1989, Astrophys. J. 342, 1187.

Cox, A. N., McNamara, B. J., and Ryan, W., 1984, Astrophys. J. 284, 250.

Cox, A. N., Morgan, S. M., Rogers, F. J., and Iglesias, C. A., 1992, Astrophys. J. $393,272$.

Dziembowski, W. A., Pamyatnykh, A. A., and Sienkiewicz, R., 1992, M.N.R.A.S., in press.

Guzik, J. A. and Cox, A. N., 1991a, Astruphys. J. 381, 333.

Guzik, J. A. and Cox, A. N., 1991 b, Delta Scuti Newsletter, ed. M. Breger, Issue 3.

Guzik, J. A. and Cox, A. N., 1992, Astrophys. J. 386, 729.

Iben, I. and MacDonald, J., 1985, Astrophys. J. 296, 540.

Korzennik, S. G., 1990, Ph. D. Dissertation, UCLA.

Libbrecht, K. G., Woodard, M. F., and Kaufman, J. M., 1990, Astrophys. J. Suppl. 74, 1129.

Matthews, J. M., 1991, Publ. Astron. Soc. Pacific 103, 5.

Michaud, G., Vauclair, G., and Vauclair, S., 1983, Astrophys. J. 267, 256.

Michaud, G. and Proffitt, C. R., 1992, in: Inside the Stars, IAU Colloquium 137, eds. A. Baglin and W. W. Weiss, Astron. Soc. of Pacific Conf. Series, in press.

Paquette, C., Pelletier, C., Fontaine, G., and Michaud, G., 1986, Astrophys. J. Suppl. 61, 177.

Poretti, E., and Antonello, E., 1988, Astron. Astrophys. 199, 191.

Proffitt, C. and Michaud, G., 1991, Astrophys. J. 380, 238.

Saez, M., Auvergne, M., Valtier, J.-C., Baglin, A., and Morel, P., 1981, Astron. Astrophys. $101,259$.

Vorontsov, S. V., Baturin, V. A., and Pamyatnykh, A. A., 1992, Nature 349, 49. 


\section{Discussion:}

M. Catelan: Could you comment on the constraints imposed on He diffusion for Population II stars by the existence of the Spite Li plateau for such objects? Isn't it true that the presence of stars at the high-temperature end of this plateau provides a strong argument for diffusion being inhibited for such stars?

J. Guzik: Diffusion can explain the Li gap in Pop I F stars, but there is no explanation yet for why diffusion doesn't also produce a Li gap in Pop II stars. This issue is discussed by Michaud and Proffitt (1991).

J.Matthews: Do the most extreme diffusion models you use to try to explain anomalous $\delta$ Scuti light curve shapes require mass loss rates comparable to what you quoted when trying to explain the period changes?

J. Guzik: Mass loss rates of $10^{-12} \mathrm{M} \circlearrowleft$ yr are required to maintain a nearly normal Pop I surface composition in the presence of diffusion. For surface $Y=0.1$, the mass loss rates can be an order of magnitude less.

A. N. Cox: I only want to say that John Percy was the first to show that helium settling can stabilize $\delta$ Scuti stars.

M. Breger: Your $\delta$ Scuti models do not include stellar rotation. Since observations show a strong inverse relation between amplitude and rotational velocity, your large amplitudes computed may not really be anomalous.

E. Antonello: I disagree with Michel (Breger). I do not think the rotational velocity is playing such an important role in $\delta$ Scuti stars, even if it known that it affects at some level the pulsational stability. Up to now, no reliable mechanism has been proposed to explain the behavior of the $\delta$ Scuti stars as regards their amplitude, and the tests with nonlinear models in the presence of inhomogeneity due to diffusion, even if their results compare only partly well with the observations, are indicating a very promising way for the interpretation of $\delta$ Scuti stars.

N. Simon: What is the accuracy of measured p-mode frequencies in the Sun, and to what extent can you simultaneously constrain the various uncertainties: mixing length, opacity, amount of diffusion, etc.?

J. Guzik: The quoted observational uncertainties for the low and intermediate degree pmodes are very small, less than $0.1 \mu \mathrm{Hz}$, so p-modes can be very effective probes of the solar structure. The mixing length is constrained by the solar radius. The adjustment of the convection zone depth to optimize the agreement between calculated and observed p-modes puts very tight constraints on the opacities at 2-5 million $\mathrm{K}$. The initial solar helium abundance is constrained by the solar luminosity, so the convection zone $\mathrm{Y}$ determined from p-modes tells us the amount of diffusion. 